\title{
Matrix Isolation of Acetonitrile Clusters from a Pulsed Supersonic Beam ${ }^{\dagger}$
}

\author{
Erich Knözinger," Peter Beichert, Jörg Hermeling, and Otto Schrems ${ }^{\ddagger}$ \\ Institut für Physikalische Chemie, Universität-GH-Siegen, Postfach 101240, D-5900 Siegen, Germany
}

Received: August 25, 1992; In Final Form: November 18, 1992

\begin{abstract}
Acetonitrile clusters $\left(\mathrm{CH}_{3} \mathrm{CN}\right)_{n}$ different from the antiparallel dimer have so far not been observed in cryogenic matrices. The combination of molecular beam and matrix isolation technique enabled us to evidence the presence of larger clusters in Ar matrix. In these experiments the task of the molecular beam is the production of the clusters via an adiabatic expansion under properly selected conditions. On the other hand, the matrix is needed for the conservation of the clusters. In combination with FT IR spectroscopy it has, therefore, essentially diagnostic relevance.
\end{abstract}

\section{Introduction}

Theoretical ${ }^{1,2}$ and experimental ${ }^{3}$ studies have definitely evidenced icosahedral structure for rare gas clusters. A characteristic property of these species is the 5-fold axis of symmetry which is strictly forbidden in equilibrium solids of rare gases (fcc, hcp). No other phenomenon manifests more impressively the dilemma of physicists and chemists devoted to the observation of the stepwise transition from isolated atoms to the condensed phase. For the transition of isolated molecules to condensed molecular matter, the situation is not fundamentally different, even though the characteristic interaction potentials raise very specific questions for each substance.

Solid acetonitrile, e.g., exhibits two thermodynamically stable phases. The high temperature modification (HTM: $P 2_{1} / c$ ) and the low temperature modification (LTM: $C m c 2_{1}$ ). In the LTM neighboring molecules have parallel orientation ${ }^{4}$ (Figure 1), whereas in the HTM nearest neighbors are antiparallel to each other ${ }^{5}$ (Figure 1). In the course of gas-phase deposition of $\mathrm{CH}_{3}-$ $\mathrm{CN}$ onto a cold target ( $13 \mathrm{~K}$ ), a solid is formed which consists exclusively of randomly oriented antiparallel dimers. ${ }^{6}$ All experimental efforts to grow larger clusters and ultimately the LTM out of these dimer units by annealing the solid definitely failed. This may be explained in terms of elementary building units of the two modifications. If at all, the antiparallel dimer may be considered as a building unit of the HTM (Figure 1). IR spectroscopic as well as X-ray diffraction studies clearly show that the solid consisting of orientationally disordered antiparallel dimers is transformed into the metastable HTM at temperatures considerably below that related to the transformation LTM $\rightarrow$ HTM $(216.9 \mathrm{~K}){ }^{6-8}$ A transformation of the solid consisting of orienta tionally disordered antiparallel dimers into the LTM would require a reorientation of $\mathrm{CH}_{3} \mathrm{CN}$ molecules by an angle of $90^{\circ}$. This is extremely unlikely at temperatures considerably below $216.9 \mathrm{~K}$. Thus the stepwise formation of the LTM by starting with one monomer and adding others successively one by one is an extremely unlikely process, too. The aggregation already stops after the formation of the antiparallel dimer, which exhibits an extraordinary high intermolecular bond energy $\left(20 \mathrm{~kJ} / \mathrm{mol}^{9}\right)$. The interaction between two such dimers is weak, since it depends essentially on quadrupole-quadrupole interaction. Similar considerations apply to the interaction between the antiparallel dimer and the monomer. On this basis phenomena observed on annealing

- To whom correspondence should be addressed.

+ Dedicated to Prof. Dr. Pedro J. Aymonino, Departamento de Química de la Facultad de Ciencias Exactas, Universidad Nacional de la Plata, Argentina, on the occasion of his 65th birthday.

I Present address: Prof. Dr. O. Schrems, Alfred-Wegener-Institut für Polar und Meeresforschung, Sektion Chemie, Postfach 120161, D-2850 Bremerhaven, Germany.

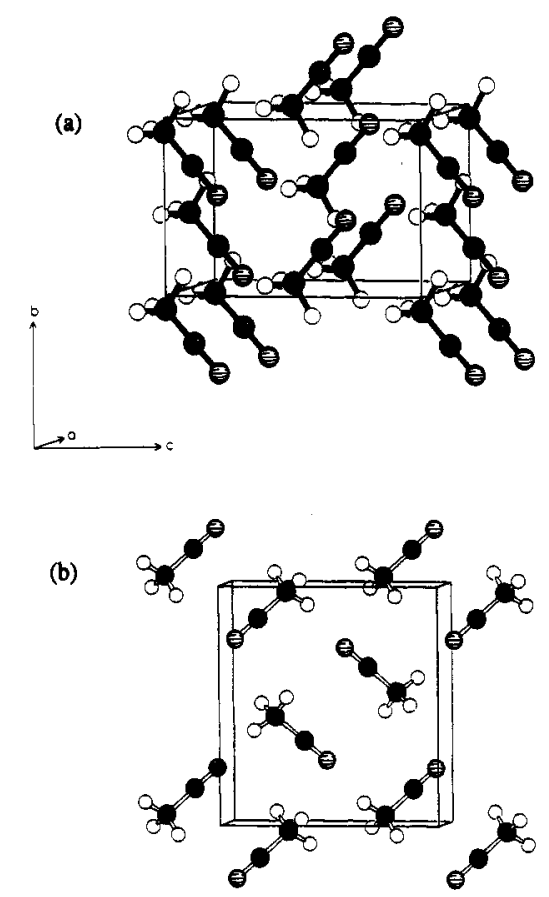

Figure 1. Structure of (a) the low temperature modification (LTM) and (b) the high temperature modification (HTM) of acetonitrile.

diverse cryogenic matrices ( $\mathrm{Ar}, \mathrm{Kr}, \mathrm{N}_{2}, \mathrm{CCl}_{4}$ ) containing monomer acetonitrile may also easily be understood. The only form of the aggregation is that of dimerization to the nonpolar species mentioned above. Other types of dimers or larger cluster species have never been detected. Thus there is so far no experimental approach which would allow one to produce the thermodynamically stable LTM of acetonitrile via the controlled formation of clusters. The respective cluster species do not originate under normal conditions such as those effective in conventional gasphase deposition or in diffusion controlled processes in solids. Special constraints have to be applied to favor the principle of cooperativity in the course of cluster formation. An adiabatic expansion appears to be particularly well suited for that purpose. Therefore, the fascinating idea of depositing cryogenic matrices and molecular solids in general from a supersonic and not from a thermal effusive molecular beam came up. Attempts to isolate molecular clusters from a free jet have been performed in the past. ${ }^{10,11}$ They have not been successful. On the other hand, only recently small size selected metal clusters produced by sputtering and subsequent quadrupole mass filtering were successfully isolated in $\mathrm{Kr}$ matrix and interrogated by fluorescence spectroscopy..$^{12}$ 


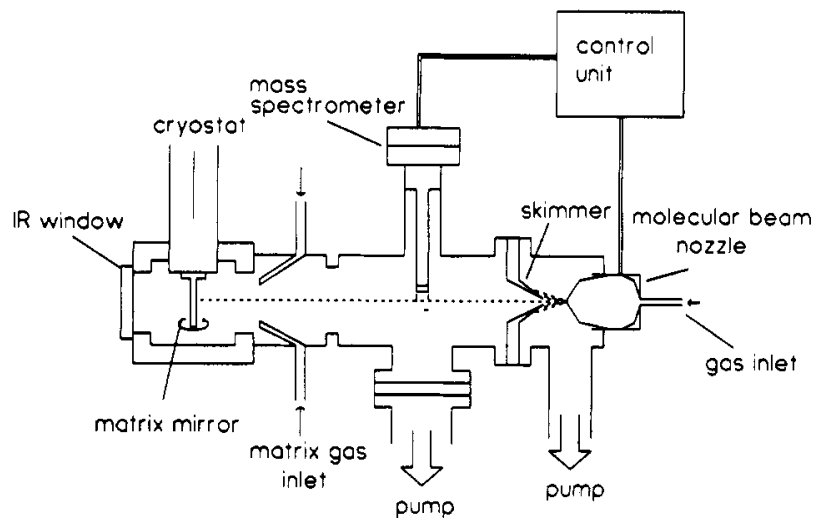

Figure 2. Schematic representation of the experimental setup which permits matrix isolation of molecular clusters produced in a pulsed supersonic beam.

\section{Experimental Section}

1. Combined Molecular Beam/Matrix Apparatus. The experimental setup of the combined molecular beam/matrix isolation apparatus is shown in Figure 2. It has to be emphasized that an intrinsic molecular beam system (pulsed supersonic valve, skimmer, differential pumping) is used here. In previous attempts ${ }^{10.11}$ a mere pulsed supersonic valve was applied which does not provide a molecular beam, but rather a so-called free jet. In view of the complexity of the processes which occur in the space between the valve orifice and the cold target, any comprehensive model of cluster formation can hardly be expected. Clusters emerging from the expansion are even likely to dissociate after collisions with thermalized species returning from the wall.

The whole apparatus consists in principle of three parts: (a) the expansion chamber, (b) the quadrupole mass spectrometer chamber, and (c) the cryostat chamber.

(a) The cubic expansion chamber (edge length $=220 \mathrm{~mm}$ ) is evacuated by an oil diffusion pump (Diff 1000, Leybold). For the molecular beam source a pulsed nozzle (type LPV 203, Lasertechnics) is used. The cylindrical orifice of the nozzle has a diameter of $0.1 \mathrm{~mm}$. The duration of a pulse is $150 \mu \mathrm{s}$; the pulse frequency amounts to $6 \mathrm{~Hz}$. During a pulse the pressure in the expansion chamber monitored by an ionization gauge increases from $2 \times 10^{-6}$ to $1 \times 10^{-4} \mathrm{mbar}$. A skimmer with an orifice of $0.53 \mathrm{~mm}$ separates the expansion chamber from the quadrupole mass spectrometer chamber.

(b) For the determination of the mass distribution of the acetonitrile clusters in the beam a quadrupole mass spectrometer (SXP 400, VG instruments) with electron impact ionization is used. It is equipped with an "open crossed beam" ion source. Therefore, it was not necessary to remove the spectrometer out of the beam during the time of matrix preparation. The whole chamber is evacuated by a turbo molecular pump (Turbovac 360 CSV, Leybold). During a pulse the pressure increases from $1 \times$ $10^{-8}$ to $5 \times 10^{-7}$ mbar.

(c) The acetonitrile clusters are deposited on a highly reflecting gold plated aluminum mirror mounted at the cold end of a closed cycle helium refrigerator (ROK 10, Leybold). The outer shroud of the cryostat is equipped with a $\mathrm{KBr}$ window to allow the IR beam to propagate through the cryogenic sample. A reflectance unit mounted in the sample compartment of the interferometer is used to reflect the beam of the light source to the cold mirror coated with the matrix. It then returns the reflected light to the spectrometer optical path. There is practically no mechanical contact between the vacuum shroud of the vibrating cryostat and the interferometer. ${ }^{13}$

It should be noted that the space between the windows in the front plate of the sample compartment and in the shroud of the cryostat has to be purged with dry air or $\mathbf{N}_{2}$ to remove traces of atmospheric water and carbon dioxide in the optical path. The
IR spectra were recorded with a Fourier transform infrared spectrometer (Model IFS 113v, Bruker Analytische Messtechnik $\mathrm{GmbH}$ ) equipped with a liquid $\mathrm{N}_{2}$ cooled $\mathrm{MCT}$ detector. A total of 100 scans were accumulated for each spectrum with a resolution of 1 wavenumber.

2. Sample Preparation. The acetonitrile clusters are produced in the course of an adiabatic expansion of acetonitrile vapour $(A)$. Before the expansion in the pulsed supersonic nozzle, neon gas $(G)$ is added. The molar ratio $A / G$ of the gas mixture is established by feeding the neon gas at a properly specified stagnation pressure (1-8 bar) through a thermostated bubbler filled with liquid acetonitrile. At a given stagnation pressure the ratio $A / G$ is then determined by the equilibrium vapor pressure of acetonitrile, i.e., by the temperature of the bubbler (223-293 $\mathrm{K})$. In fact, the deposition of the acetonitrile clusters occurs from a seeded beam. The temperature of the mirror $(14 \mathrm{~K})$ where the acetonitrile clusters are deposited does not permit the condensation of the seed gas neon which has to be pumped off efficiently. In order to isolate the clusters, a thermal effusive stream of argon as matrix gas is directed from the matrix gas inlet onto the mirror (Figure 2). The argon gas flow is 0.05 $\mathrm{mmol} / \mathrm{h}$. From the total amount of $\mathrm{Ar}$ and $\mathrm{CH}_{3} \mathrm{CN}$ consumed in the course of matrix preparation, we roughly estimate the molar ratio of $\mathrm{CH}_{3} \mathrm{CN}$ in $\mathrm{Ar}$ as being of the order of $1: 1000$. The preparation time of a matrix is typically $14 \mathrm{~h}$.

\section{Concepts of Evaluation of Mass and IR Spectra}

1. Mass Spectra. In order to be able to compare the mass distributions in the cluster beam for different expansion conditions, the intensity of the mass peaks had to be normalized. This is accomplished by dividing the integral intensity of each peak by the sum of the integral intensities of all cluster ions $(41,42,83$, $124,165, \ldots \mathrm{amu})$. The resulting values are essentially independent of the total molar amount of $\mathrm{CH}_{3} \mathrm{CN}$ which flows per time unit through the supersonic valve. Fragments related exclusively to intramolecular bond ruptures ( $<41 \mathrm{amu}$ ) do not contribute additional information with respect to the mass distribution of the cluster ions and, therefore, have not been taken into account.

2. IR Spectra. In condensed phase, the intrinsic line widths are often greater than the distance between two neighboring bands. In this case resolution enhancement of the instrument does not lead to a separation of the bands. Fourier self-deconvolution (FSD) provides a way of computationally resolving them ${ }^{14}$ (Figure 3a).

For this procedure we assume, that the profile of each absorption band is Lorentzian

$$
A(\tilde{\nu})=\frac{A_{0} \gamma^{2}}{\gamma^{2}+\left(\tilde{\nu}-\tilde{\nu}_{0}\right)^{2}}
$$

where $A_{0}$ is the maximum absorbance of the band, $\tilde{\nu}_{0}$ is the wavenumber for $A_{0}$ and $\gamma$ is the half-width at half-height. The decision for Lorentzians was not dictated by any concepts related to dynamic processes (dephasing, vibrational energy relaxation, etc.) in the matrix but by merely practical reasons. Lorentzians permit a more efficient separation of the constituents of a complex band. Our studies clearly show that both the number and the positions of the resulting subbands are essentially independent of whether Lorentzians or Gaussians were applied in the FSD procedure.

The cosine Fourier transform of $A(\tilde{\nu})$ is given by

$$
\begin{aligned}
I(x) & =F T\{A(\tilde{\nu})\}=\int_{0}^{\infty} A(\tilde{\nu}) \cos (2 \pi \tilde{\nu} x) \mathrm{d} \tilde{\nu} \\
& =0.5 A_{0} \gamma \cos \left(2 \pi \tilde{\nu}_{0} x\right) \exp (-2 \pi \gamma x)
\end{aligned}
$$

where $x$ has the dimension of a length $(\mathrm{cm})$.

The cosine term in eq 2 depends on the position $\nu_{0}$ of the band, while the exponential decay term is determined by $\gamma$. Therefore, 

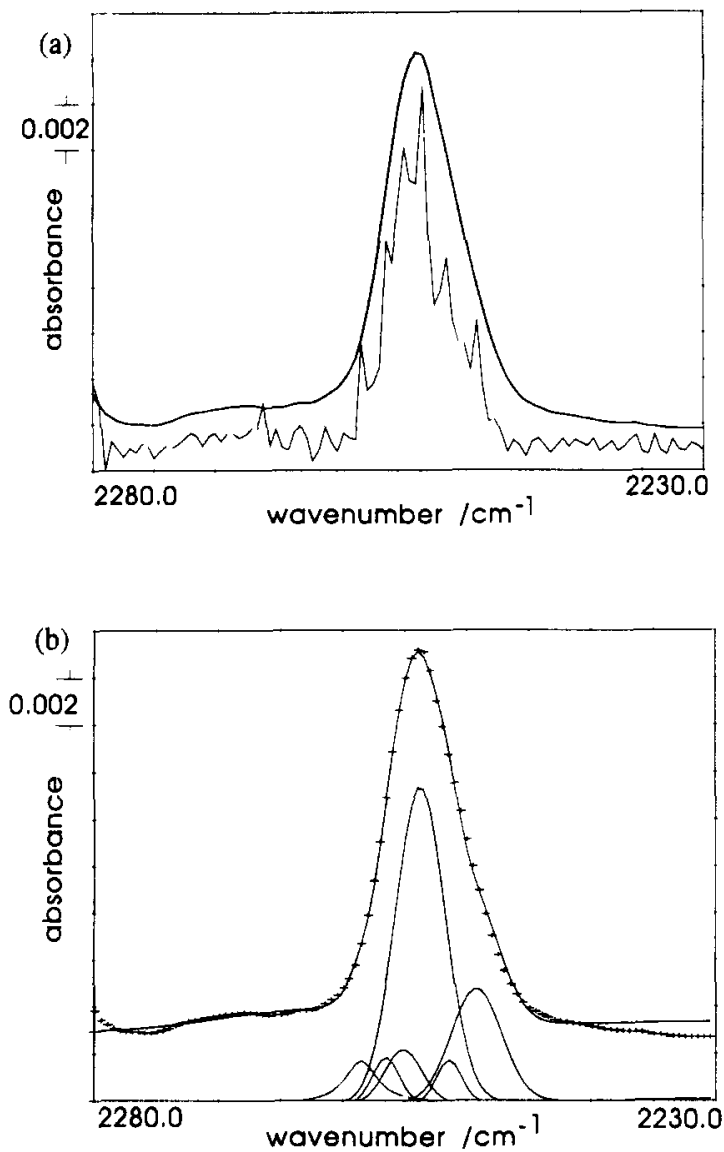

Figure 3. (a) Fourier self-deconvolution (FSD) of the complex $\nu_{C} \equiv N$ band related to an acetonitrile cluster size distribution in Ar matrix (expansion conditions prior to matrix deposition: $\mathrm{CH}_{3} \mathrm{CN} / \mathrm{Ne}=1: 100$; stagnation pressure, 5 bar). The triangular appodization function applied led to a bandwidth reduction by a factor of 3. (b) Band fitting based on the six band positions obtained in a. The fit parameters are the integral intensity values of these six subbands. For further details see Concepts of Evaluation.

with a decrease of the rate of decay of the exponential, the width of the band is reduced. FSD is performed by multiplying eq 2 by $\exp (2 \gamma \pi x)$ and then by replacing the exponential term by an appropriate apodization function $D(x)$ which decays more slowly. This leads to a new function

$$
\begin{aligned}
I^{\prime}(x) & =I(x) \exp (2 \pi \gamma x) D(x) \\
& =0.5 A_{0} \gamma \cos \left(2 \pi \tilde{\nu}_{0} x\right) D(x)
\end{aligned}
$$

Reverse Fourier transformation $\mathrm{FT}^{-1}$ provides a band $A^{\prime}(\tilde{\nu})$ with reduced bandwidth. Its shape is given by the Fourier transform of the apodization function $D(x)$.

Thus the efficiency of the described computational resolution enhancement considerably depends on what the function $D(x)$ looks like. The limiting factor of the procedure is the $S / N$ ratio in the input spectrum. A detailed description of FSD is given elsewhere. ${ }^{14,15}$ Figure 3 a shows-as an example-the application of the FSD to the $\nu_{C}=\mathrm{N}$ band observed for a certain size distribution of acetonitrile clusters isolated in an Ar matrix. Without any further information this essentially unstructured band would certainly not be a favorable case for application of FSD with all its known pitfalls and risks. The reliability of the procedure has to be checked by varying the experimental conditions of cluster production and then controlling the stability of the subband positions with respect to these variations (see also Discussion). In addition, one should try to confirm as many of these band positions as possible by separate experiments.

The subbands resulting from FSD of the $\nu_{\mathrm{C}=\mathrm{N}}$ band are then fitted with Gaussian and Lorentzian curves according to a least squares procedure (Figure 3b). The centers of the curves are
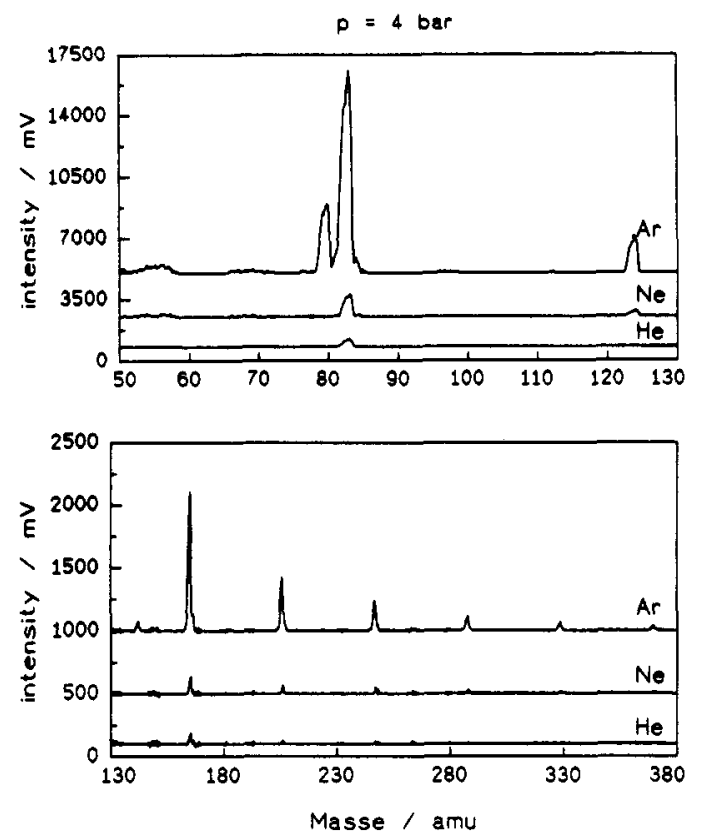

Figure 4. Mass spectra of the supersonic acetonitrile cluster beam seeded with different rare gases $\mathrm{G}=\mathrm{He}$, Ne, Ar. Expansion conditions: $\mathrm{CH}_{3} \mathrm{CN} / \mathrm{G}=1: 100$; stagnation pressure $=4$ bar.

taken from FSD. Four of them coincide with the values of the monomer, the dimer, the high temperature modification (HTM), and the low temperature modification (LTM) (Table IV). These values were determined in additional experiments which also provided the respective full width at half-maximum (fwhm) values (Table IV). It should be pointed out that IR band widths of molecular clusters isolated in rare gas matrices are, in general, considerably larger than those observed for isolated small molecules [e.g. 16]. Thus values of $3-7 \mathrm{~cm}^{-1}$ as presented in Table IV are not unusual. Even careful annealing does not contribute very much to a sharpening of the bands. Some uncertainty is related to the fwhm of the two remaining species. The respective values were obtained by fitting.

The procedure described enables us to fit all spectra obtained under most different expansion conditions (stagnation pressure, 1-8 bar; concentration, 1:1000 to 1:25).

\section{Results}

The mass spectra of seeded beams of acetonitrile in rare gas ( $\mathrm{He}, \mathrm{Ne}, \mathrm{Ar} ; 1: 100)$ exhibit a progression of mass peaks MP (Figure 4) regularly spaced at a distance of $M_{\mathrm{CH}_{3} \mathrm{CN}}=41 \mathrm{amu}$, the molar mass of the acetonitrile monomer,

$$
\mathrm{MP}=n M_{\mathrm{CH}_{3} \mathrm{CN}}+M_{\mathrm{H}}
$$

where $n=1,2,3, \ldots$ is the degree of aggregation and $M_{\mathrm{H}}=1$ amu is the molar mass of the hydrogen atom. Evidently the mass spectra reflect cluster formation to an extent far beyond that observed for a corresponding static mixture. ${ }^{17}$ No clusters other than dimers have ever been detected before in the static gas phase ${ }^{17}$ or in cryogenic matrices. 6.13 .18 The mass peak positions (eq 4) in Figures 4 and 5 differ from those of simple clusters $\left(\mathrm{CH}_{3} \mathrm{CN}\right)_{n}$ by $1 \mathrm{amu}$. This is obviously the result of fragmentation initiated by electron impact ionization. Molecular fragments are observed in the mass interval from 1 to 41 amu (see Concepts of Evaluation). The intensity of the cluster peaks increases with the molar mass of the rare gas used in the expansion from He to Ar (Figure 4). In spite of this observation Ne was preferred to $\mathrm{Ar}$ in seeded beams of acetonitrile, since self-aggregation and formation of heteroclusters are more likely for $\mathrm{Ar}$ than for $\mathrm{Ne}$ (melting points of $\mathrm{Ar}$ and $\mathrm{Ne}, 83.7 \mathrm{~K}$ and $24.5 \mathrm{~K}$, respectively). Therefore, the presence of $\mathrm{Ar}$ in the expansion was expected to augment the 


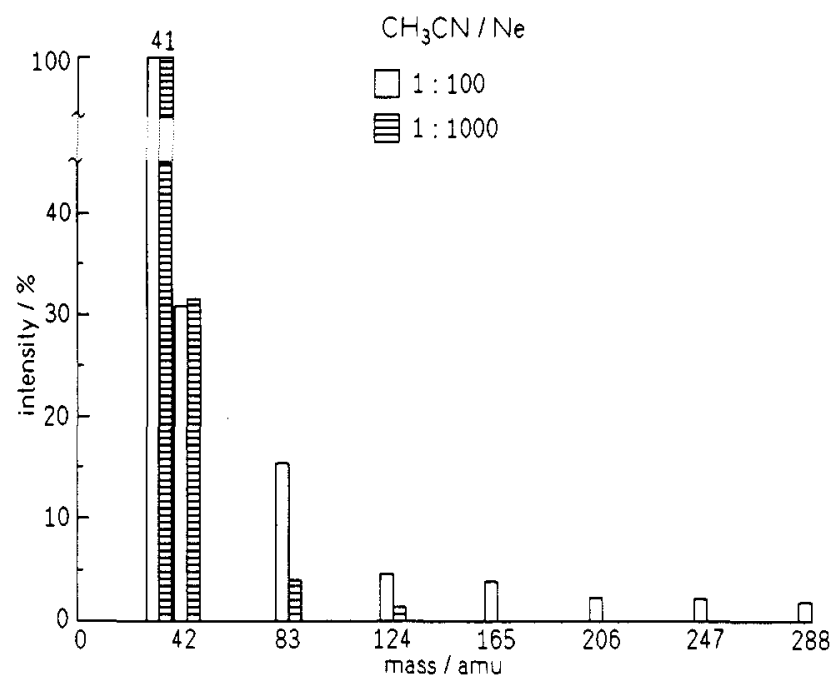

Figure 5. Influence of the concentration $\left(\mathrm{CH}_{3} \mathrm{CN} / \mathrm{Ne}=1: 1000,1: 100\right)$ on the mass spectra in the supersonic acetonitrile cluster beam seeded with $\mathrm{Ne}$ (stagnation pressure $=5$ bar).

variety of different cluster species considerably. This would scramble the IR spectra and complicate their evaluation.

As Figures 5 and $6 \mathrm{a}, \mathrm{b}$ demonstrate, the intensity of the cluster mass peaks sensitively depends on both the stagnation pressure and the concentration of $\mathrm{CH}_{3} \mathrm{CN}$ in the seeded beam. As shown in Figure 2, the molecular beam is finally mixed with an $\mathrm{Ar}$ stream added through the matrix gas inlet. The matrix mirror mounted at the cold end of the cryostat is kept at a temperature of $14 \mathrm{~K}$ which permits the $\mathrm{CH}_{3} \mathrm{CN}$ cluster to be trapped in an Ar matrix. Under these conditions Ne remains quantitatively in the gas phase and is pumped away. The resulting IR spectra are strongly influenced by the expansion conditions of the seeded beam (stagnation pressure, concentration of $\mathrm{CH}_{3} \mathrm{CN}$ in $\mathrm{Ne}$ ). This is particularly true for the band contour of the $\mathrm{CN}$ stretching vibration which is significantly altered with the concentration of $\mathrm{CH}_{3} \mathrm{CN}$ (Figure 7). Similar effects are observed on varying the stagnation pressure. Fourier self-deconvolution provides six subbands, the positions of which are essentially independent of the expansion conditions (Table I) and of the type of band contour used in the procedure (Lorentzian/Gaussian). For all experiments listed in Table I the band contour of the $\mathrm{CN}$ stretching band can be fitted satisfactorily by varying the integral intensity values of these six or even less than six subbands. Thus the observed $\mathrm{CN}$ stretching band may be represented by a linear combination of at most six constituents, the set of the corresponding coefficients being intimately related to the expansion conditions. The trend followed by these coefficients as a function of the concentration of $\mathrm{CH}_{3} \mathrm{CN}$ in $\mathrm{Ne}$ at a stagnation pressure of 5 bar (Figure 8) is physically reasonable: with an increase in the concentration of acetonitrile in the beam seeded with $\mathrm{Ne}$ the smaller particles are consumed in favor of the larger ones. Table II evidences, in a more general way, that the fit procedure is not trivial. At low values of stagnation pressure and concentration, only the three components at higher wavenumbers (corresponding to small species) are required. For high values of the variables describing the expansion conditions, the three low wavenumber constituents (larger cluster) nearly suffice for fitting the band. Thus six amplitudes are really needed as parameters to fit the band shape for all expansion conditions.

\section{Discussion}

The efficiency of $\mathrm{CH}_{3} \mathrm{CN}$ cluster formation in seeded supersonic beams significantly increases with the molar mass of the rare gas (Figure 4). This may be understood in terms of two different physical principles: (a) Under the same expansion conditions the rare gas with smaller molar mass attains the larger maximum velocity in the beam. ${ }^{19}$ This velocity is finally adopted also by the $\mathrm{CH}_{3} \mathrm{CN}$ molecules. Therefore, in a $\mathrm{CH}_{3} \mathrm{CN}$ beam seeded with $\mathrm{He}$ or $\mathrm{Ne}$, the expansion zone where collisions between $\mathrm{CH}_{3-}$ $\mathrm{CN}$ molecules take place is traversed faster than that in a $\mathrm{CH}_{3-}$ $\mathrm{CN}$ beam seeded with Ar. (b) The momentum transfer from the seed gas to $\mathrm{CH}_{3} \mathrm{CN}$ molecules and the related cooling effect for $\mathrm{CH}_{3} \mathrm{CN}$ is more efficient for $\mathrm{Ar}\left(M_{\mathrm{Ar}}=40 \mathrm{amu}, M_{\mathrm{CH}_{3} \mathrm{CN}}=41\right.$ amu) than for $\mathrm{He}$ and $\mathrm{Ne}\left(M_{\mathrm{He}}=4 \mathrm{amu}, M_{\mathrm{Ne}}=20 \mathrm{amu}\right) .{ }^{20} \mathrm{This}$ also favors $\mathrm{CH}_{3} \mathrm{CN}$ cluster formation in Ar. On the other hand $\mathrm{Ar}$ is much more likely than $\mathrm{Ne}$ to undergo aggregation by itself (self-aggregation as well as formation of heteroclusters with $\mathrm{CH}_{3} \mathrm{CN}^{21}$ ). Having deposited an acetonitrile beam seeded with $\mathrm{Ar}$, the contribution of $\mathrm{Ar}$ to cluster formation leads to such a variety of local environments of the $\mathrm{CH}_{3} \mathrm{CN}$ molecules in the solid mixture that an evaluation of the IR spectra in terms of a set of $\mathrm{CH}_{3} \mathrm{CN}$ clusters $\left(\left(\mathrm{CH}_{3} \mathrm{CN}\right)_{n}\right.$ with $\left.n=2,3,4, \ldots\right)$ is not possible (see discussion below). The mass spectra (Figure 4) do not provide significant evidence for clusters of the type (Ar) ${ }_{n}$ and $(\mathrm{Ar})_{n}\left(\mathrm{CH}_{3} \mathrm{CN}\right)_{m}$. However, the appearance of these species in the mass spectra is most likely suppressed by previous electron impact ionization.

Both Figures 4 and 5 clearly show that the acetonitrile clusters originating from the adiabatic expansion are also subjected to fragmentation initiated by electron impact ionization. The experimental evidence is provided by the appearance of the progression of cluster mass peaks for the ions of the type $\left(\mathrm{CH}_{3}\right.$ $\mathrm{CN}{ }_{n} \mathrm{H}^{+}$(eq 5a). Buck et al.22 (see also ref 23) derived the fragmentation channels presented in eqs $5 \mathrm{a}-\mathrm{d}$. These results

$$
\begin{aligned}
& \mathrm{CH}_{3} \mathrm{CN} \stackrel{\theta^{-}}{\longrightarrow} \mathrm{CH}_{3} \mathrm{CN}^{+}+2 e^{-}
\end{aligned}
$$

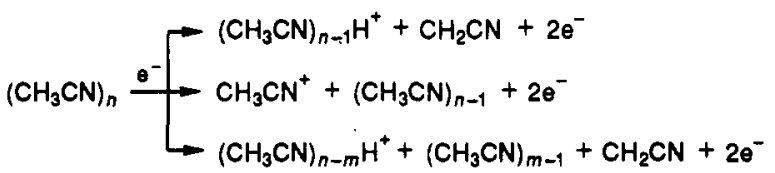

were based on mass spectroscopic studies of mass selected $\mathrm{CH}_{3}$ $\mathrm{CN}$ cluster beams after electron impact ionization. Table III provides some quantitative aspects of the fragmentation process. For small clusters $(n=2,3,4)$ electron impact ionization initiates mainly the formation of protonated acetonitrile molecules $\left(\left(\mathrm{CH}_{3}\right.\right.$ $\mathrm{CN}) \mathrm{H}^{+}$). As the degree of aggregation $n$ increases, the percentage of this species with respect to the total amount of ions originating from the fragmentation of $\left(\mathrm{CH}_{3} \mathrm{CN}\right)_{n}$ decreases (see $n=3$ and $n=4$ in Table III). On the other hand the percentage of protonated clusters $\left(\left(\mathrm{CH}_{3} \mathrm{CN}\right)_{n} \mathrm{H}^{+}\right.$with $\left.n \geq 2\right)$ and of molecular cations $\mathrm{CH}_{3} \mathrm{CN}^{+}$increases within the same series. On the basis of these results the dependence of the mass peak intensities on the overall concentration of acetonitrile in $\mathrm{Ne}$ and on the stagnation pressure may easily be understood (Figure 6a,b). At very low concentrations monomer molecules prevail in the beam. Their percentage decreases with increasing concentration of acetonitrile because of the formation of dimers, trimers, etc. This explains the intensity decrease of the mass peak 41 with increasing concentration in the range below $1 \mathrm{~mol} \% \mathrm{CH}_{3} \mathrm{CN}$ in Ne at a stagnation pressure of 2.2 bar (Figure 6a, top). With a further increase in the concentration, larger clusters are formed. Their fragmentation mechanisms (eqs $5 c$,d) favor the formation of the molecular cation $\left(\mathrm{CH}_{3} \mathrm{CN}^{+}\right)$. This is in perfect agreement with the shape of the curve in Figure 6a(top), which exhibits an increase in intensity for the mass peak 41 with increasing concentration in the range above $1 \mathrm{~mol} \% \mathrm{CH}_{3} \mathrm{CN}$ in $\mathrm{Ne}$. By an increase of the stagnation pressure from 2.2 to 5 bar (Figure 6b, top), the curve in Figure 6a(top), in particular its maximum, is shifted to smaller values of the $\mathrm{CH}_{3} \mathrm{CN}$ concentration. The higher stagnation pressure necessarily implies more collisions in the expansion zone and, therefore, allows the acetonitrile molecules to cool more efficiently. This may be interpreted in terms of an increased 

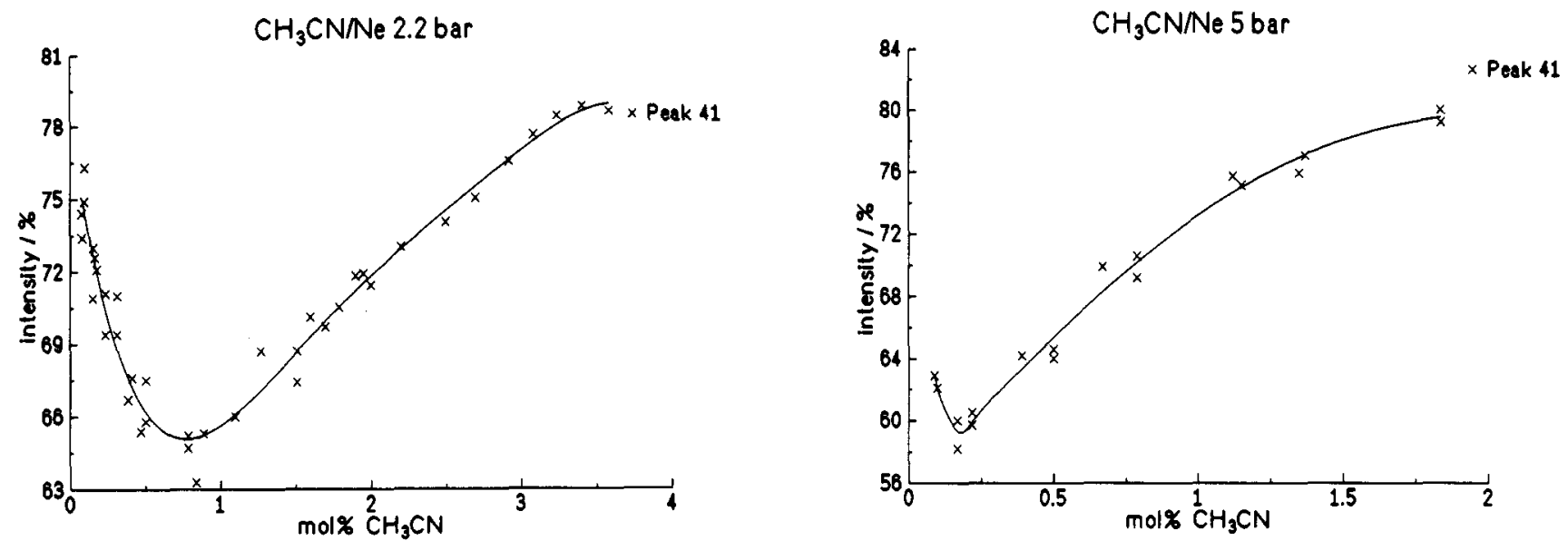

(a)

(b)
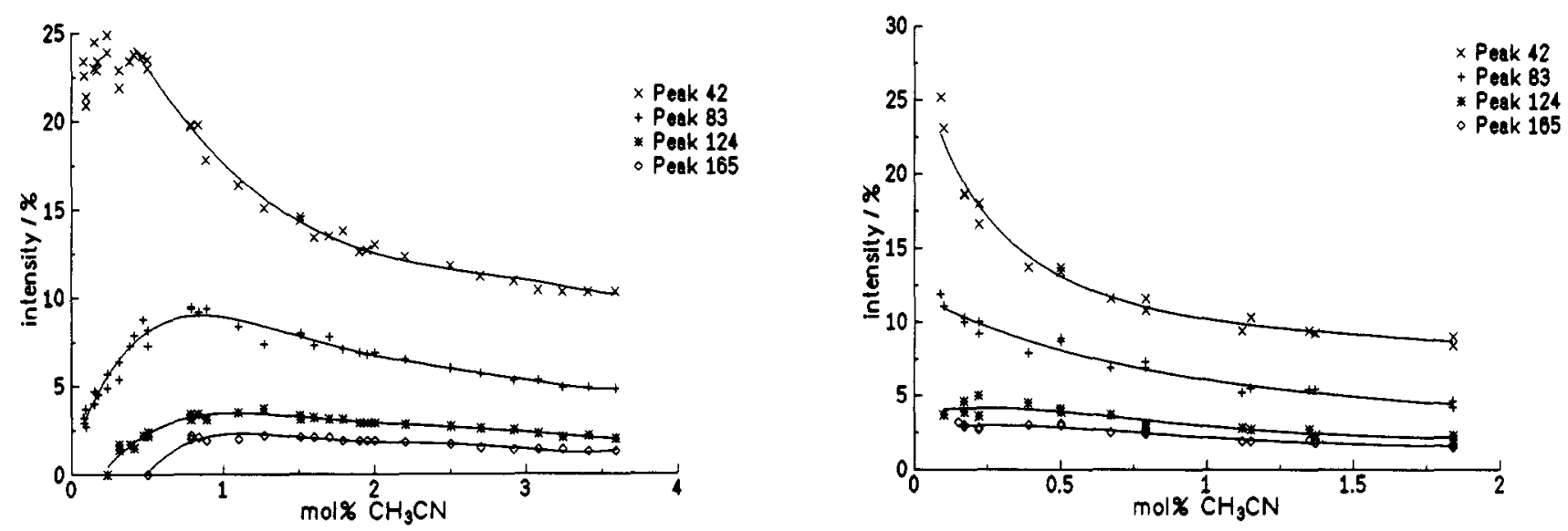

Figure 6. Influence of the concentration $\left(\mathrm{CH}_{3} \mathrm{CH} / \mathrm{Ne}\right)$ on the intensity of individual mass peaks (MP $\left.=41,42,83,124,165\right)$ for different stagnation pressures: (a) 2.2 bar; (b) 5.0 bar.

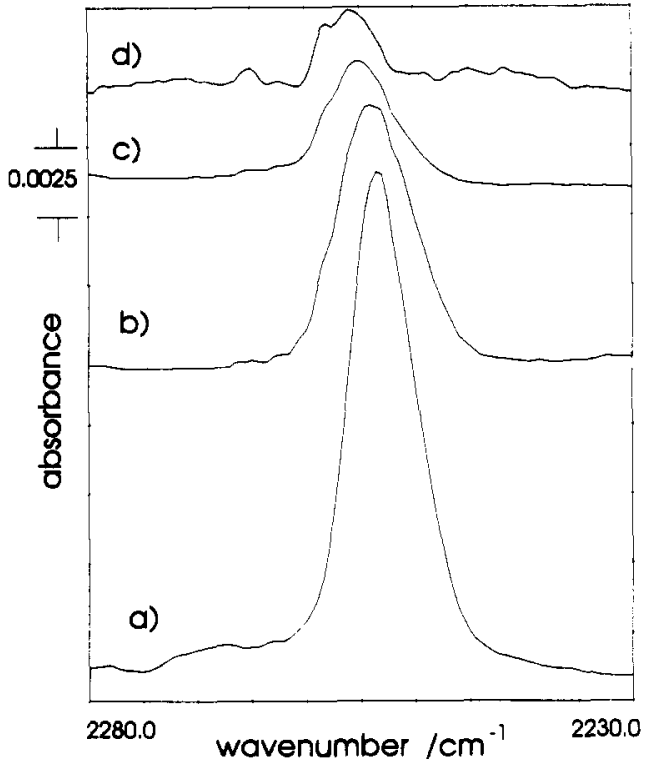

Figure 7. $v_{\mathrm{C}=\mathrm{N}}$ band of acetonitrile deposited from a seeded supersonic beam together with a thermal effusive Ar stream. Deposition temperature: $13 \mathrm{~K}$. Deposition rate (Ar): $0.05 \mathrm{mmol} / \mathrm{h}$. Stagnation pressure: 5.0 bar. (a) $\mathrm{CH}_{3} \mathrm{CN} / \mathrm{Ne}=1: 50$; (b) $\mathrm{CH}_{3} \mathrm{CN} / \mathrm{Ne}=1: 100$; (c) $\mathrm{CH}_{3} \mathrm{CN} /$ $\mathrm{Ne}=1: 200 ;$ (d) $\mathrm{CH}_{3} \mathrm{CN} / \mathrm{Ne}=1: 1000$.

clustering efficiency and explains the shift of the curve in Figure $6 \mathrm{~b}$ (top) with respect to that in Figure 6a(top).

The mass peak 42 is mainly the result of fragmentation of small clusters as dimers, trimers, etc. (Table III). Their percentages have a maximum at medium acetonitrile concen- trations. This is reflected by the respective curve in Figure 6a(bottom). Similar considerations explain the traces for the intensity of the mass peaks 83,124 , and 165 , which represent the cluster fragments for $n \geq 3, n \geq 4$, and $n \geq 5$, respectively (Figure $6 \mathrm{a}$, bottom). The increase of the stagnation pressure ( 5 bar in Figure 6b, 2.2 bar in Figure 6a) shifts the maxima to lower concentration values, such that they can no longer be detected (Figure 6b, bottom).

Without any doubt the adiabatic expansion of gaseous mixtures of $2 \mathrm{~mol} \mathrm{\%} \mathrm{CH}_{3} \mathrm{CN}$ or less in Ne permits the formation of molecular clusters of acetonitrile with $n \geq 2$. To the best of our knowledge such species have never been observed in static gasphase samples. ${ }^{10}$ As mentioned above, $\mathrm{CH}_{3} \mathrm{CN}$ molecules oppose the formation of trimers and larger clusters in cryogenic matrices and in gas-phase-deposited pure solids. $6,8,13$ Therefore, we decided to isolate the acetonitrile clusters, produced in a molecular beam after adiabatic expansion, in a cryogenic matrix, e.g., in an Ar matrix. This should permit the IR spectroscopic observation of the $\mathrm{CH}_{3} \mathrm{CN}$ trimers and larger species at a limited level of experimental expenditure. An alternative would be the IR laser spectroscopic analysis of $\mathrm{CH}_{3} \mathrm{CN}$ clusters in the molecular beam. It requires bolometric detection and tunable laser techniques. ${ }^{24}$

As stated in the previous discussion the adiabatic expansion does not provide size-selected acetonitrile clusters, but rather a broad size distribution (Figures 4 and 5). The shape of this distribution certainly depends on the expansion conditions. There is, however, no chance to tune it such that one cluster size would prevail. Buck's technique of size selection by He scattering ${ }^{23,24}$ is beyond the level of experimental sophistication which we can afford. In addition, it has to be doubted whether FT IR spectroscopy is sensitive enough to cope with the extremely low 
TABLE I: Results of Fourier Self-Deconvolution Applied to the $v_{\mathrm{C} \equiv \mathrm{N}}$ Band Observed after Deposition of Acetonitrile Clusters Isolated in an Argon Matrix from a Supersonic Beam Seeded with Neon

\begin{tabular}{|c|c|c|c|c|c|c|c|}
\hline \multirow{2}{*}{$\frac{p / \text { bar }}{1.0}$} & \multirow{2}{*}{$\frac{\text { molar ratio } \mathrm{CH}_{3} \mathrm{CN} / \mathrm{Ne}}{1: 1000}$} & \multicolumn{6}{|c|}{ band position $/ \mathrm{cm}^{-1}$} \\
\hline & & 2258.1 & 2256.0 & 2254.5 & & & \\
\hline 1.0 & $1: 200$ & 2258.3 & 2256.0 & 2254.5 & 2253.1 & & \\
\hline 1.0 & $1: 100$ & 2258.0 & 2256.0 & 2254.7 & & & \\
\hline 1.0 & $1: 50$ & 2258.2 & 2256.2 & 2254.6 & 2253.2 & & \\
\hline 1.0 & $1: 25$ & 2258.1 & 2256.0 & 2254.6 & 2253.1 & & \\
\hline 2.2 & $1: 1000$ & 2258.3 & 2256.0 & 2254.6 & & & \\
\hline 2.2 & $1: 200$ & 2258.3 & 2256.0 & 2254.5 & 2253.1 & 2251.0 & \\
\hline 2.2 & $1: 100$ & 2258.0 & 2256.1 & 2254.8 & 2253.3 & 2251.3 & \\
\hline 2.2 & $1: 50$ & 2257.9 & 2256.1 & 2254.7 & 2253.2 & 2250.9 & \\
\hline 2.2 & $1: 25$ & 2257.9 & 2256.0 & 2254.7 & 2253.5 & & \\
\hline 3.5 & $1: 200$ & 2258.1 & 2256.1 & 2254.7 & 2253.0 & & \\
\hline 3.5 & $1: 100$ & 2257.6 & 2256.1 & 2254.7 & 2253.2 & & \\
\hline 3.5 & 1:35 & & 2256.6 & 2254.6 & 2253.2 & 2251.3 & 2248.5 \\
\hline 5.0 & $1: 1000$ & 2258.5 & 2256.1 & 2254.7 & 2253.0 & 2250.8 & \\
\hline 5.0 & $1: 200$ & 2258.5 & 2256.1 & 2254.7 & 2253.1 & 2250.9 & 2248.9 \\
\hline 5.0 & $1: 100$ & 2258.1 & 2256.1 & 2254.7 & 2253.0 & 2251.0 & 2248.8 \\
\hline 5.0 & 1:50 & 2258.1 & 2256.1 & 2254.7 & 2253.3 & 2251.2 & 2248.5 \\
\hline 6.5 & $1: 200$ & 2258.1 & 2256.1 & 2254.7 & 2253.5 & 2251.5 & \\
\hline 6.5 & $1: 100$ & & 2256.4 & 2254.7 & 2253.2 & 2251.3 & 2248.5 \\
\hline 8.0 & $1: 100$ & 2257.9 & 2256.5 & 2254.7 & 2253.5 & 2251.3 & 2248.9 \\
\hline
\end{tabular}

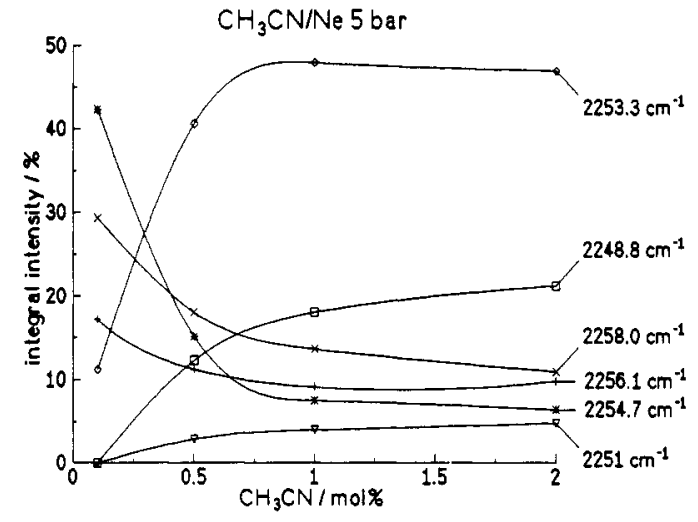

Figure 8. Influence of the concentration of acetonitrile in the supersonic beam $\left(\mathrm{CH}_{3} \mathrm{CN} / \mathrm{Ne}\right)$ on the integral intensity of the six subbands which constitute the $\nu_{C}=N$ band (compare Figure 3 and Figure 7) after isolation of the acetonitrile clusters in Ar matrix (stagnation pressure, 5 bar).

yield in size-selected $\mathrm{CH}_{3} \mathrm{CN}$ cluster beams. Thus the size distribution of $\mathrm{CH}_{3} \mathrm{CN}$ clusters after adiabatic expansion was a matter of fact to which we had to resign ourselves.

Previous FT IR studies of $\mathrm{CH}_{3} \mathrm{CN}$ and $\left(\mathrm{CH}_{3} \mathrm{CN}\right)_{2}$ in $\mathrm{Ar}$ matrix ${ }^{18,25}$ and of the two solid modifications (HTM and LTM) ${ }^{7,8}$ clearly show that the $\mathrm{CN}$ stretching mode $\left(\nu_{\mathrm{C}=\mathrm{N}}\right)$ sensitively reacts on environmental changes (Table IV). In addition, it exhibits a strong extinction coefficient and is not subjected to complications related to degeneracy. Therefore, we concentrated ourselves on $\nu_{\mathrm{C}=\mathrm{N}}$ as probe for the size distribution of acetonitrile clusters isolated in Ar after their production in an adiabatic expansion. Obviously it is not possible to detect separate bands for the diverse cluster species. The band contour observed (Figure 7) depends, however, as sensitively on the expansion conditions as the mass distribution in the beam (Figure $6 a, b$ ). In order to extract specific information related to different cluster species from the band contour, Fourier self-deconvolution (see Concepts of Evaluation) was applied. It provided six band positions (Table I), four of them being in extraordinary good agreement with those of $\mathrm{CH}_{3}$ $\mathrm{CN}$ and $\left(\mathrm{CH}_{3} \mathrm{CN}\right)_{2}$ and of the two solid modifications (Table IV). The variation of the stagnation pressure by a factor of 8 and of the concentration of acetonitrile by nearly 2 orders of magnitude does not change significantly the resulting band positions (Table 1). This stability clearly emphasizes the reliability of the procedure. We adopt, therefore, the bands around 2254.7 and $2253.2 \mathrm{~cm}^{-1}$ as being related to clusters of intermediate size such as trimers, tetramers, or/and pentamers.
The integral intensity values of the six subbands are proportional to the respective concentrations of the related cluster species in the matrix. It is, however, not possible to derive a size distribution owing to the unknown absorbance coefficients of $\nu_{C} \equiv N$ for these clusters. On the other hand, the dependence of the respective integral intensity values on the total concentration of acetonitrile in the gas mixture subjected to adiabatic expansion has a clear physical meaning: It describes the trend in the occurrence of each of the cluster species as the concentration of acetonitrile in the gas mixture is increased. Thus, in Figure 8 (stagnation pressure, 5 bar) a decrease for the smaller species (bands at 2258.1 2256.1 , and $2254.7 \mathrm{~cm}^{-1}$ ) and an increase for the larger ones (bands at 2253.3,2251.0, and $2248.8 \mathrm{~cm}^{-1}$ ) are observed. This is a consistent picture which is in agreement with the expectation: increasing the concentration of acetonitrile in the initial gas mixture shifts the cluster size distribution to larger values, i.e., favors the consumption of small species and the formation of larger ones. Insofar there is agreement between the IR (Figure 8 and Table II) and the MS data (Figure 6a,b), in particular, if we consider the fragment of the mass 41 (Figure 6a,b, top). For the larger fragments the shape of the curves in Figure $6 a, b$ (bottom) is significantly influenced by the complicated fragmentation mechanisms (eqs $5 c, d$ ). The attempt to establish a correlation between these MS and the IR data would essentially involve speculation. One should not forget that, in the paper presented here, mass spectroscopy has mainly diagnostic relevance: it is needed to optimize the working conditions of the pulsed supersonic valve and the expansion conditions for cluster formation in the molecular beam.

Different from the matrix studies presented here, clusters exhibiting the structure of the LTM have not been observed in molecular beam experiments. ${ }^{22}$ However, it is possible that nonequilibrium clusters formed in the process of adiabatic expansion relax into an equilibrium-like structure in the course of condensation together with Ar gas.

Experiments with Ar as seed gas instead of Ne (Figure 4) did not permit an evaluation of the IR spectra obtained after deposition of the respective molecular beam with Ar. FSD did not provide stable positions of the subbands. This must somehow be related to the fact that the molecular beam contains pure Ar and pure $\mathrm{CH}_{3} \mathrm{CN}$ clusters and, in addition, mixed species of the type $\mathrm{Ar}_{m}\left(\mathrm{CH}_{3} \mathrm{CN}\right)_{n}$. This huge variety of different cluster species probably leads to a similar variety of different sites for acetonitrile molecules after deposition of the molecular beam. The elucidation of such a system is certainly beyond the scope of both FSD and band fitting. 
TABLE II: Relative Integral Intensity Values Resulting from the Fit Procedure for Different Expansion Conditions

\begin{tabular}{|c|c|c|c|c|c|c|c|}
\hline \multirow[b]{3}{*}{ stagnation pressure/bar } & \multirow[b]{3}{*}{ molar ratio $\mathrm{CH}_{3} \mathrm{CN} / \mathrm{Ne}$} & \multicolumn{6}{|c|}{ relative integral intensity $/ \%$} \\
\hline & & \multirow{2}{*}{$\begin{array}{c}\text { monomer } \\
2258.1\end{array}$} & \multirow{2}{*}{$\begin{array}{l}\text { dimer } \\
2256.1\end{array}$} & \multicolumn{2}{|c|}{ so far unknown } & \multirow{2}{*}{$\begin{array}{l}\text { HTM } \\
2251.0\end{array}$} & \multirow{2}{*}{$\begin{array}{l}\text { LTM } \\
2248.8\end{array}$} \\
\hline & & & & 2254.7 & 2253.3 & & \\
\hline 1 & $1: 1000$ & 68 & 26 & 6 & & & \\
\hline 5 & $1: 1000$ & 28 & 17 & 43 & 12 & & \\
\hline 5 & $1: 100$ & 13 & 9 & 8 & 47 & 5 & 18 \\
\hline 8 & $1: 100$ & 5 & 3 & 6 & 52 & 10 & 24 \\
\hline
\end{tabular}

TABLE III: Fragmentation Pattern of Acetonitrile Clusters Observed in Mass Selected Cluster Beams by Buck et al. ${ }^{22}$

\begin{tabular}{ccccc}
\hline & \multicolumn{4}{c}{ relative intensity of the fragment peaks $/ \%$} \\
\cline { 2 - 5 } cluster & $\begin{array}{c}\mathrm{CH}_{3} \mathrm{CN}^{+} \\
(41 \mathrm{amu})\end{array}$ & $\begin{array}{c}\left(\mathrm{CH}_{3} \mathrm{CN}\right) \mathrm{H}^{+} \\
(42 \mathrm{amu})\end{array}$ & $\begin{array}{c}\left(\mathrm{CH}_{3} \mathrm{CN}\right)_{2} \mathrm{H}^{+} \\
(83 \mathrm{amu})\end{array}$ & $\begin{array}{c}\left(\mathrm{CH}_{3} \mathrm{CN}\right)_{3} \mathrm{H}^{+} \\
(124 \mathrm{amu})\end{array}$ \\
\hline $\mathrm{CH}_{3} \mathrm{CN}$ & 100 & & & \\
$\left(\mathrm{CH}_{3} \mathrm{CN}\right)_{2}$ & 37 & 63 & & \\
$\left(\mathrm{CH}_{3} \mathrm{CN}\right)_{3}$ & 23 & 69 & 8 & \\
$\left(\mathrm{CH}_{3} \mathrm{CN}\right)_{4}$ & 24 & 47 & 28 & 1
\end{tabular}

TABLE IV: Band Positions and Fwhm of the CN Stretching Vibration in the IR Spectra of Diverse Acetonitrile Species

\begin{tabular}{|c|c|c|c|}
\hline & & $\begin{array}{c}\mathrm{C} \equiv \mathrm{N} \text { stretch } / \\
\mathrm{cm}^{-1}\end{array}$ & $\begin{array}{c}\mathrm{fwhm} / \\
\mathrm{cm}^{-1}\end{array}$ \\
\hline known species & $\begin{array}{l}\text { monomer in } \text { gas }^{7} \\
\text { monomer in } \mathrm{Ar}^{a} \\
\text { dimer in } \mathrm{Ar}^{a} \\
\mathrm{HTM}^{8} a \\
\mathrm{LTM}^{7 a}\end{array}$ & $\begin{array}{l}2267.0 \\
2258.0 \\
2256.1 \\
2251.0 \\
2248.8\end{array}$ & $\begin{array}{l}3.2 \\
3.8 \\
3.6 \\
6.9\end{array}$ \\
\hline so far unknown species & & $\begin{array}{l}2254.7 \\
2253.3\end{array}$ & $\begin{array}{l}5.1 \\
6.8\end{array}$ \\
\hline
\end{tabular}

a This work.

So far the IR spectra were interpreted tacitly assuming that there is one IR active $C N$ stretching vibration for each species. In fact, this is not correct. For a cluster consisting of $n \mathrm{CH}_{3} \mathrm{CN}$ molecules there are $n$ modes with characteristic phase differences between neighboring molecules. Out of these modes one single is IR active for the monomer-this is trivial-and for the perfectly ordered crystal-this is due to the $k=0$ selection rule. For clusters of intermediate size $(n=2,3, \ldots) n$ IR active modes should be observed, provided symmetry selection rules are not effective. They are, however, closely spaced, and one of them is always of dominating intensity. This was shown for growing linear chains of $\mathrm{HCN}$ molecules. In this case the modes exhibiting maximum IR intensity are the in-phase vibrations. ${ }^{26}$ Therefore, the assumption of one single band per cluster species appears to be a justified approximation for the evaluation of the IR spectra.

The stepwise transition from isolated molecules to the solid phase was intensively studied in our group for HCN. One aspect of this research was cited above. ${ }^{26}$ Another important result was that already at an early stage of aggregation the vibrational properties of HCN clusters resemble those of the respective solid phase. Six different small species (hydrogen bonded linear chains) from the monomer to-most likely-the hexamer could be discriminated on the basis of the in-phase librations. All larger chains contribute exclusively to the $k=0$ librational mode of the thermodynamically stable solid phase. For acetonitrile (dipoledipole forces) the intermolecular pair interactions are weaker than those for hydrogen cyanide (hydrogen bonding). Therefore, the spread in frequency for the normal modes of all species from the isolated monomer to the solid phase is less efficient for $\mathrm{CH}_{3}$ $\mathrm{CN}$ than for $\mathrm{HCN}$. The wavenumber interval in which the $\nu_{\mathrm{C} \equiv \mathrm{N}}$ bands of all possible acetonitrile species isolated in Ar appear comprises only $10 \mathrm{~cm}^{-1}$ (Table IV). For other intramolecular normal vibrations it is even smaller. Thus the limited number of acetonitrile clusters (6; see Figures 3 and 8 as well as Table I) observed in Ar marix by IR spectroscopy should not surprise. It is a matter of fact that IR quanta are not able to discriminate hexamers or heptamers from infinitely large clusters provided the building principle is the same. This means that the $k=0$ selection rule already applies to cluster species of very limited size.

The kinetic energy of the acetonitrile clusters in the supersonic beam is given by eq 6 where $n$ is the degree of aggregation and $v_{\max , \mathrm{Ne}}$ is the maximum velocity of $\mathrm{Ne}$ atoms in the beam. Far distant from the expansion zone, collisions between equal and unequal species $\left(\mathrm{Ne},\left(\mathrm{CH}_{3} \mathrm{CN}\right)_{n}\right.$ are extremely unlikely. This

$$
E_{\text {kin. }}=1 /{ }_{2} n M_{\mathrm{CH}_{3} \mathrm{CN}} v_{\max , \mathrm{Ne}^{2}}
$$

necessarily implies that the acetonitrile clusters adopt the maximum velocity of the $\mathrm{Ne}$ atoms which-to a first approximation-exclusively depends on the temperature of the pressurized gas mixture in the reservoir feeding the pulsed supersonic valve. The resulting kinetic energy of the dimer is of the same order of magnitude as the intermolecular bonding energy. Provided there is an efficient energy transfer from the translation of the dimer to the respective reaction coordinate, fragmentation can take place in the deposition process. For larger clusters the kinetic energy is correspondingly greater-proportional to the degree $n$ of aggregation. However, the number of motional degrees of freedom is increased by an even larger factor. This leads to a reduction of the probability of energy transfer from translation to an appropriate reaction coordinate and, therefore, to a reduction of fragmentation. Another influence which counteracts cluster fragmentation in the course of deposition stems from the addition of Ar gas (Figure 2). As Ar is added in excess, many collisions between $\mathrm{Ar}$ and $\left(\mathrm{CH}_{3} \mathrm{CN}\right)_{n}$ take place immediately before the deposition. On the other hand, the momentum transfer from $\left(\mathrm{CH}_{3} \mathrm{CN}\right)_{n}$ to $\mathrm{Ar}$ is limited owing to the considerable differences of the respective molar masses. In an intuitive picture the addition of Ar gas may, therefore, be considered as a procedure which favors soft landing of the clusters on the cold matrix support. To cut a very complicated and so far speculative story short: cluster fragmentation during the deposition may not be excluded, but it is-to the best of our present knowledge-not a likely process.

\section{V1. Conclusion}

IR spectroscopy easily lends itself to the study of aggregated species in static systems, such as gas cells and cryogenic matrices. However, it is in general not possible to trace the stepwise transition from the isolated molecules to the condensed molecular phase. On the other hand, the whole manifold of clusters emerges from a supersonic orifice into a molecular beam. In seeded beams the characterization of molecular clusters by IR spectroscopy suffers from insufficient sensitivity (FT IR) and limited free spectral range of tunable IR lasers (bolometric detection). As shown in this paper, the advantage of conventional FT IR and molecular beam technique can be combined by trapping the molecular clusters from a seeded supersonic beam in a cryogenic matrix. In this way the presence of acetonitrile clusters which have never been observed before in matrices or in static gas phase could be evidenced. There is, however, a severe limitation. The clusters cannot be produced selectively. They rather exhibit a more or less broad size distribution which is reflected by the band contour related to the fundamental vibration under consideration. The 
variation of the expansion conditions permits band contours to be obtained which cannot be created by any linear combination of the bands related to so far known species: monomers, dimer, and crystal-like particles which had previously been detected in matrices emerging from thermal effusive jets or in pure condensed phases, respectively. On the other hand the adiabatic expansion in a pulsed supersonic valve is only a necessary, but not a sufficient, condition for the deposition and isolation of molecular clusters on the cold target. Diverse previous attempts to evidence cluster formation in a matrix deposited from a free jet have definitely failed. Obviously the collision free conditions of molecular clusters in a superconic molecular beam are required, if these species are to be trapped in a cryogenic matrix as described in this paper.

Acknowledgment. This research was funded by the Deutsche Forschungsgemeinschaft, the Minister für Wissenschaft und Forschung des Landes NRW, the Fonds der Chemischen Industrie, and the Kanzler der Universität Siegen. We are grateful for the considerable financial support. The technical support of Mr.D. Gaumann and Mr. G. Neumann in setting up the combined molecular beam/matrix isolation apparatus (Figure 2) is gratefully acknowledged. Thanks are also due to Dr. N. Ernsting (Max-Planck-Institut für biophysikalische Chemie, Göttingen) for providing a skimmer and to Prof. U. Buck (Max-PlanckInstitut für Stőmungsforschung, Göttingen), Prof. G. Scoles (University of Princeton), and Dr. H. Selzle (Technische Universität München) for valuable advice and recommendations concerning the molecular beam equipment.

\section{References and Notes}

(1) Lethbridge, P. G.; Del Mistro, G.; Stace, A. J. J. Chem. Phys. 1990, 93, 1995

(2) Franke, G.; Schulte, J. Z. Phys. D 1989, 12, 65.
(3) Ding, A.; Hesslich, J. Chem. Phys. Lett. 1983, 94, 54.

(4) Antson, O. K.; Tilli, K. J.; Andersen, N. H. Acta Crystallogr. 1987, $B 43,296$.

(5) Barrow, M. Acta Crystallogr. 1981, B37, 2239.

(6) Langel, W.; Kollhoff, H.; Knözinger, E. Ber. Bunsen-Ges. Phys. Chem. $1985,89,927$

(7) Pace, E. L.; Noe, L. J. J. Chem. Phys. 1968, 49, 5317.

(8) Milligan, D. E.; Jacox, M. E. J. Mol. Spectrosc. 1962, 8, 126.

(9) Böhm, H. J.; Ahlrichs, R.; Scharf, P.; Schiffer, H. J. Chem. Phys. 1984, 81 (3), 1389 .

(10) Pimentel, G. C. University of California, Berkely, personal communication, 1983.

(11) Jones, L. H.; Ekberg, S. A.; Swanson, B. I. J. Chem. Phys. 1985, 82, 1055 .

(12) Harbich, W.; Fedrigo, S.; Meyer, F.; Lindsay, D. M.; Lignieres, J.; Rivoal, J. C.; Kreisle, D. J. Chem. Phys. 1990, 93, 8535.

(13) Knözinger, E.; Schrems, O. In Vibrational Spectra and Structure; Elsevier: Amsterdam, 1987; Vol. 16.

(14) Kauppinen, J. K.; Moffat, D. J.; Mantsch, H. H.; Cameron, D. G. Appl. Spectrosc. 1981, 35, 271.

(15) Kauppinen, J. K.; Moffat, D. J.; Mantsch, H. H.; Cameron, D. G. Appl. Opt. 1981, 20, 1866.

(16) Schrems, O.; Huth, M.; Kollhoff, H.; Wittenbeck, R.; Knözinger, E. Ber. Bunsen-Ges. Phys. Chem. 1987, 91, 1261.

(17) Leutloff, D.; Knözinger, E. Spectrosc. Lett. 1979, /2, 815

(18) Freedman, T. B.; Nixon, E. R. Spectrochim. Acta 1972, A28, 1375.

(19) Kappes, M.; Kunz, R. W.; Schumacher, E. Chem. Phys. Lett. 1982. $91(6), 413$

(20) Miller, D. R. In Atomic and Molecular Beam Methods; Oxford University Press: New York, Oxford, U.K., 1988, Vol. I, Pp 41-44.

(21) Gough, T. E.; Knight, D. G.; Scoles, G. Chem. Phys. Lett. 1983, 97,

(22) Buck, U. Max-Planck-Institut für Strömungsforschung, Göttingen, Germany, personal communication, 1991.

(23) Buck, U.; Gu, X. J.; Krohne, R.; Lauenstein, Ch. Chem. Phys, Lett. 1990, I74, 247.

(24) Levandier, D. J.; Mengel, M.; McCombie, J.; Scoles, G. In The chemical physics of atomic and molecular clusters, VIIth; Enrico Fermi School: Varenna, Italy, 1988.

(25) Huth, M.; Knözinger, E. Unpublished. 3430 .

(26) Langel, W.; Kollhoff, H.; Knŏzinger, E. J. Chem. Phys. 1989, 90 , 